\title{
JARDINES PRIVADOS Y CONSUMO DE AGUA EN LAS PERIFERIAS URBANAS DE LA COMARCA DE LA SELVA (GIRONA)
}

\author{
Xavier Garcia ${ }^{1}$, Anna Ribas² y Albert Llausàs ${ }^{3}$ \\ Departamento de Geografía. Universitat de Girona ${ }^{1}$ \\ Departamento de Geografía. Universitat de Girona ${ }^{2}$ \\ Department of Resource Management and Geography. The University of Melbourne ${ }^{3}$
}

\section{RESUMEN}

En los espacios residenciales de baja densidad, la tipología de jardín se convierte en un factor determinante del consumo de agua en el hogar. En este artículo se utiliza el análisis clúster para identificar las diferentes tipologías de jardín privado que se dan en las urbanizaciones del sur de la comarca de La Selva (Girona). La información de base se obtuvo de 240 encuestas realizadas en viviendas unifamiliares de urbanizaciones ubicadas en 9 municipios de la comarca. Los resultados muestran la existencia de 4 tipologías de jardín: ornamental, huerto, césped y arbolado. A continuación se describen las relaciones existentes entre los distintos perfiles sociodemográficos de sus propietarios y las diversas tipologías de jardines identificadas. Por último, un análisis de regresión logística reveló cómo cada patrón de jardín está claramente asociado con unas determinadas características sociodemográficas de las familias, como serían la edad de los residentes, su interés en la jardinería, el tamaño de la familia o el tipo de ocupación (permanente/temporal) de la vivienda.

Palabras clave: jardines privados, urbanización de baja densidad, consumo de agua, variables sociodemográficas, Girona.

\section{ABSTRACT \\ Private landscaping and water consumption in suburban developments in La Selva County (Girona)}

In the suburban low-density areas, the landscaping typology becomes a relevant factor in regards to the average domestic water consumption per household. Firstly, in this study we applied a cluster analysis in order to find out the main landscaping typologies in this specific urban context in La Selva County. We used the information from a survey of 240 households, in the suburban low-density areas of 9 of the municipalities in this county. Results show 4 main garden typologies: ornamental, vegetable, lawn and tree. The study also intends to explore how the landscaping patterns obtained in the previous analysis are associated with particular sociodemographic characteristics of the households. Furthermore, a multinomial logistic regression analysis revealed how each landscaping pattern is associated with particular sociodemographic characteristics of the households, e.g., age, garden interest, household size or type of residential occupation.

Key words: Private landscaping, suburban low-density areas, water consumption, sociodemographic variables, Girona.

\section{INTRODUCCIÓN}

Para hacer frente a los problemas relacionados con la escasez de agua (Collins et al., 2009), son muchos los países y regiones del mundo que deben buscar nuevas fuentes alternativas de abastecimiento (como serían la reutilización y desalinización del agua) y, paralelamente, implantar acciones de gestión a fin de aumentar la eficiencia en el uso de agua (Thomas y Durham, 2003).

Contacto: Xavier Garcia: xavier.garciaacosta@udg.edu; Anna Ribas: anna.ribas@udg.edu; Albert Llausàs: albert.llausas@udg.edu 
En Europa, y no sólo en el arco mediterráneo, estudios recientes muestran como la escasez de agua es un problema cada vez más común (EEA, 2009). Además, los pronósticos no son nada favorables, dado el futuro aumento en la frecuencia de sequías, a la par que se prevé un incremento del tamaño de la población (Collins et al., 2009). Ante tal escenario, la preocupación sobre la disponibilidad de agua en la cantidad y calidad suficientes ha convertido en más urgente aún la necesidad de avanzar hacia un enfoque de sostenibilidad en la planificación y gestión de los recursos hídricos. En dicho enfoque, la estrategia de gestión centrada en la demanda tiene un papel muy relevante. Es un ejemplo de ello el informe publicado recientemente bajo el título de Blueprint to Safeguard Europe's Water Resources que tiene como objetivo plantear acciones para proteger los recursos hídricos europeos a partir de una amplia evaluación de las políticas actuales entorno a estos recursos (EEA, 2012). En este informe se destaca como un eje prioritario para alcanzar tal objetivo la aplicación de medidas de gestión orientadas al incremento de la eficiencia en los diferentes usos del agua.

En Cataluña, esta preocupación por alcanzar un conocimiento exhaustivo de la naturaleza y características de la demanda de agua para usos domésticos o urbanos ha recabado el interés de varios estudios (Saurí, 2003; Domene y Saurí, 2006; Garcia et al., 2013a, Garcia et al., 2013b). Obtener este conocimiento ayudará a apuntar medidas de gestión del agua centradas en la eficiencia en su uso. La bibliografía científica apunta al desarrollo del modelo territorial urbanístico de baja densidad como la principal causa del aumento en el consumo de agua para usos domésticos. El principal impacto de la expansión de este tipo de espacio urbano respecto a los consumos de agua en las viviendas es la aparición de usos externos al hogar con fines recreativos, como las piscinas, los jardines o la horticultura (Saurí, 2003).

La reciente y creciente expansión del modelo urbanístico de baja densidad en el litoral mediterráneo español se viene desarrollando de forma completamente ajena a las repercusiones que implica este modelo en la demanda y consumo de agua (EEA, 2006). Por lo tanto, la comprensión de cómo los propietarios de estos espacios urbanos forman y mantienen su jardín privado tiene importantes implicaciones dado que, en un ambiente con escasez hídrica como el litoral mediterráneo peninsular, la tipología de jardín puede llegar a ser un factor muy relevante para la gestión hídrica. Cabe recordar que el riego del jardín, al igual que el llenado de la piscina, se consideran usos del agua discrecionales, hecho que los sitúa en el punto de mira de las administraciones públicas cuando se requiere realizar restricciones del consumo de agua en momentos de sequía (sirva de ejemplo el Decreto 84/2007 de sequía del Gobierno Catalán) o diversas campañas de sensibilización ambiental que se han llevado a término en los últimos años).

\subsection{Patrones de ajardinamiento y factores sociodemográficos asociados}

Diferentes razones pueden motivar a los residentes en viviendas unifamiliares a tener un jardín en la parte exterior de su vivienda. Una casa con jardín comporta, por ejemplo, el aumento del valor de venta de la vivienda (Syme et al., 1980) o el aumento del sentido de pertenencia a un lugar (Sime, 1993). También se ha convertido cada vez más en una actividad de recreación, a la par que puede jugar una importante función en cuanto a las relaciones de uno mismo con la familia y sus amistades (Bhatti y Church, 2000) o puede resultar un elemento clave en términos de tendencia actual de presentación social, sobre todo en las partes más visibles del jardín (Larsen y Harlan, 2006).

En general, la elección de una determinada tipología de jardín depende de una diversidad de factores concurrentes. Hurd (2006), en un estudio realizado en tres ciudades del estado de Nuevo México (EE. UU.), demostró que la superficie del jardín que se ocupaba con césped estaba directamente relacionada con factores como el precio del agua, el nivel educativo y el grado de concienciación de los residentes sobre la necesidad de ahorrar agua. Cuanto más elevado era el precio del agua menor era el porcentaje de césped presente en el jardín y mayor la superficie ocupada por otros elementos de menor requerimiento hídrico (por ejemplo, especies vegetales con bajas necesidades de riego, como determinadas especies de arbustos o árboles, plantas crasas, además de rocas, etc.).

Las condiciones socioeconómicas de los hogares también influyen en el tipo de jardín que se escoge (Larson et al., 2009). El jardín privado puede ser visto como un elemento residencial orientado a comunicar el propio poder adquisitivo y su identidad dentro de la comunidad (Larsen y Harlan, 2006). 
Por lo tanto, dado el coste elevado que requiere en mantenimiento y riego, es habitual que los propietarios más adinerados sean más propensos a preferir césped en sus jardines (Larson et al., 2009). Este patrón se ejemplifica en la Región Metropolitana de Barcelona, donde el césped es el elemento vegetal más importante en superficie en los jardines privados de los hogares con mayores ingresos. En cambio, en el caso de familias de clase media, el arbusto mediterráneo es el elemento en superficie más común (Domene y Saurí, 2003). Larsen y Harlan (2006), en su estudio llevado a cabo en Phoenix (EE.UU.), llegan a la conclusión que los propietarios de bajos ingresos tienden a preferir un jardín con césped en la parte frontal de la vivienda, mientras que los propietarios de rentas medias optan por un jardín donde domina la vegetación nativa (desértica), y los de mayores ingresos se inclinan por el jardín de vegetación nativa o del tipo "oasis"1. Hope et al. (2003) encontraron, también en Phoenix, una relación directa entre el nivel de riqueza del área donde se ubicaba el hogar y la diversidad de vegetación del jardín, de tal manera que los hogares de mayores rendas iban asociados a paisajes urbanos con una mayor diversidad de plantas. Los autores bautizaron este fenómeno como "efecto lujo" (en inglés luxury effect). Otro estudio realizado por Mustafa et al. (2010) en San Petersburgo (también en EE.UU.), aportó resultados contradictorios, ya que la tendencia a optar por jardines más xéricos (modelo opuesto al césped) era independiente del nivel socioeconómico de los hogares, pero no así de su nivel educativo. Hurd (2006) mostró resultados similares, puesto que encontró que el nivel educativo moderaba el deseo de poner césped en el jardín y contribuía decisivamente en la elección de jardines con pocos requerimientos hídricos.

La disposición espacial de las viviendas también juega un papel crucial en la determinación de los patrones de ajardinamiento. Este efecto sería más importante en la parte más visible del jardín - en general, la parte delantera de la vivienda - más orientado a seguir los estándares de conformidad social, en contraste con la parte posterior de la misma, más orientada al ocio y al tiempo libre (Larsen y Harlan, 2006). Así lo constatan Zmyslony y Gagnon (2000) en un estudio realizado, a escala de sección de calle, en Montreal (Canadá). Los autores encontraron que la distancia (proximidad) y similitud de cada vivienda con las que se encontraban a su alrededor, además de otros parámetros estructurales, hacia aumentar la similitud de los jardines delanteros de las casas dentro de la misma sección de calle.

En un estudio realizado en jardines privados de la ciudad de Mesa (EE.UU.), Yabiku et al. (2008) demostraron que uno de los factores que determinaban la preferencia por un jardín xérico era el género, siendo el femenino el que menos valoraba la opción xérica. En relación con estos resultados, Davidson y Freudenburg (1996) argumentaron que las mujeres, en comparación con los hombres, son generalmente más propensas a preocuparse por los peligros que amenazan a sus familias. Este mismo argumento podría aclarar porque Yabiku et al. también encontraron que los hogares con hijos valoraban positivamente los jardines mésicos (entendido como el tipo de jardín antagónico al xérico), puesto que serían vistos como el espacio ideal de recreo sin riesgos para los niños. Larson et al. (2009), refiriéndose a los mismos resultados sobre la presencia de niños en el hogar y su relación con la tipología de jardín, argumentaron en la misma línea que los padres con hijos podrían percibir los jardines xéricos cómo potencialmente más peligrosos:

"Los progenitores pueden percibir que los jardines con plantas del desierto, con plantas con pinchos y rocas en el suelo, son menos adecuados en comparación a los jardines con césped". (Larson et al., 2009, p. 923)

Sin embargo, otros estudios no siempre han encontrado una relación significativa entre la presencia de niños y jardines con mucho césped (Hurd, 2006).

Van den Berg y Van Winsum-Westra (2010) en su estudio realizado para un conjunto de ciudades holandesas, constataron que los residentes de mayor edad preferían mayoritariamente los jardines con un césped esmeradamente cuidado a los de tipo más silvestre o romántico. Esta observación podría estar relacionada, según estos autores, con el hecho de que el envejecimiento se asocia a una mayor "necesidad personal de estructura" (ver Hess, 2001) y, en consecuencia, a que las personas mayores prefieran este tipo de jardín (Van den Berg y Van Winsum-Westra, 2010).

1 Jardín con plantas con flores de colores vivos y vegetación exuberante. La mayoría de las plantas son especies exóticas y su elevada densidad recrea un ambiente tropical. Este tipo de jardín también requiere ser regado diariamente. 
Head et al. (2004) estudiaron como los antecedentes culturales de los diferentes grupos étnicos que emigraron a Australia condicionaban el tipo de jardinería que se practicaba en sus viviendas. En los patios de los residentes procedentes de Macedonia y Vietnam, abundaba la producción de hortalizas y árboles y arbustos frutales. Los jardines de los originarios de Inglaterra eran más variados: mientras algunos preferían plantar flores ornamentales no nativas, otros se decantaban por las plantas nativas.

Por último, junto con la conformidad social y los factores espaciales que influyen en los patrones de ajardinamento, otras variables de carácter psicológico y culturales pueden contribuir a explicar qué tipo de jardinería se prefiere o se practica en un hogar. Así, en otro estudio llevado a cabo en la ciudad de Las Cruces (EE.UU.), se comprobó que los residentes que albergaban más conocimiento sobre las plantas del jardín mostraban una mayor preferencia por la presencia de plantas no autóctonas (St. Hilaire et al., 2003). Los valores ambientales de los residentes también influyen en el tipo de jardinería que se acaba practicando puesto que hay una relación positiva entre el nivel de concienciación ambiental y el compromiso personal por practicar una jardinería más ecológica (con menores necesidades de riego, entre otras características) (Kiesling y Manning, 2010). Yabiku et al. (2008) demostraron como las actitudes y creencias medioambientales se relacionaban con un rechazo significativo a la opción por el jardín mésico. Larson et al. (2010) encontraron que los residentes que preferían los jardines tipos "oasis" eran, con respecto a la orientación de sus valores, personas claramente antropocéntricas en comparación con aquellas con jardines totalmente mésicos o xéricos. Sin embargo, una orientación claramente biocéntrica conducía a la elección de un tipo de jardín en el que dominaba el césped.

\section{2. ÁREA DE ESTUDIO}

El área de estudio comprende 9 municipios situados en la parte más meridional de las comarcas gerundenses, más concretamente, la parte sur de la comarca de La Selva. Blanes, Lloret de Mar y Tossa de Mar son municipios litorales mientras que Vidreres, Sils, Maçanet de la Selva, Caldes de Malavella, Vilobí d'Onyar y Santa Coloma de Farners constituyen el resto de municipios del área de estudio. Los 130.000 habitantes censados en 2012 pueden llegar a superar los 200.000 durante los fines de semana y, especialmente, coincidiendo con la temporada de vacaciones debido a los propietarios de segundas residencias y a los turistas que la visitan.

Figura 1. Localización de la comarca de La Selva y de las urbanizaciones del área de estudio.

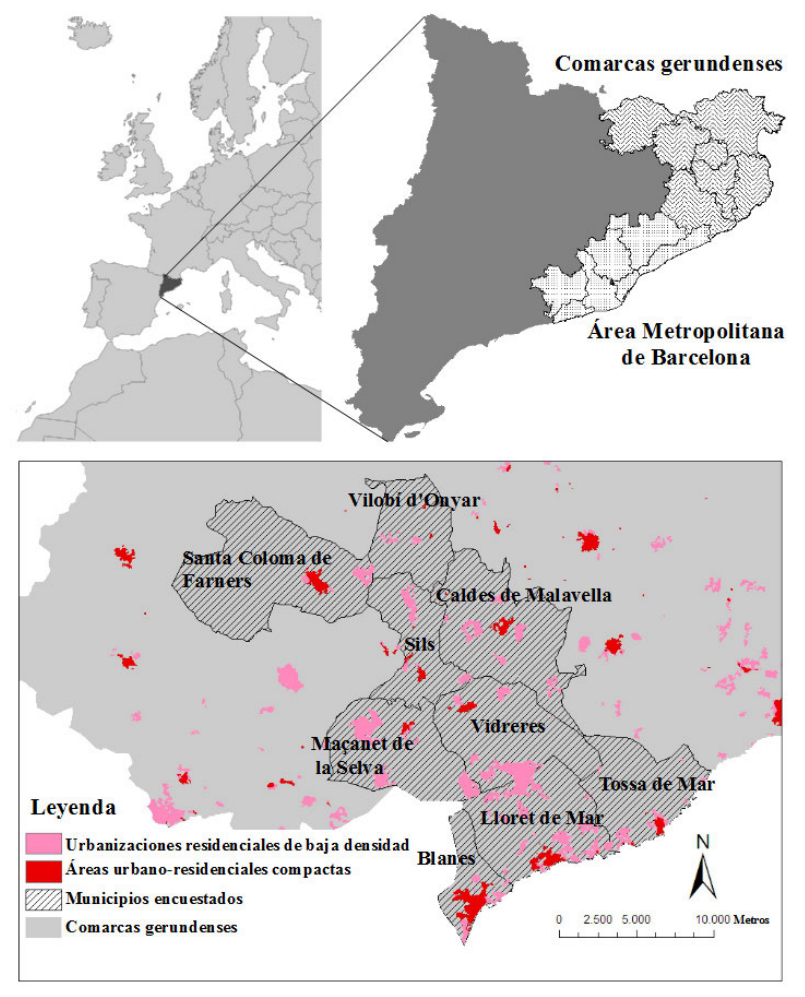


Dada su situación litoral o de segunda línea respecto a la costa y su proximidad con el Área Metropolitana de Barcelona (AMB), estos municipios (y la comarca en general) se caracterizan por configurar un área donde, a lo largo de estos últimos decenios, se ha dado un proceso claro de metropolitanización bajo la influencia de la AMB (Nel.lo, 2001). Mientras que originalmente las relaciones metropolitanas con este territorio se centraban en el turismo y la ubicación de segundas residencias, actualmente se ha convertido en una extensión de la propia $\mathrm{AMB}$, tanto en ubicación de actividades económicas como por fijación de primeras residencias (DTS, 2010).

No es de extrañar que la proliferación de urbanizaciones en esta zona ( $y$ con ellas el modelo de vivienda unifamiliar con jardín) responda, en un primer momento (décadas de 1960 y 1970), a la demanda de segundas residencias por parte de habitantes en la AMB, el Área Urbana de Girona y, también en el caso de las urbanizaciones de los municipios litorales, de países europeos como Francia o Alemania. Esta tipología de urbanización se inicia primero en áreas forestales del litoral y prelitoral y en zonas a menudo de gran pendiente. Las de primera línea de costa, a menudo ubicadas próximas a calas, se distinguen por agrupar viviendas de alto standing vinculadas al turismo europeo o de clases altas. En contraste, las urbanizaciones de interior fueron edificadas muchas veces de forma caótica y simple, con elementos del paisaje urbano (aceras, instalaciones eléctricas, asfaltaje de las calles, etc.) de baja calidad o incluso inexistentes. Este tipo de espacios se han beneficiado de la demanda creciente de viviendas unifamiliares por parte de capas sociales más amplias de raíz urbana procedentes tanto de la AMB como de las principales ciudades de las comarcas gerundenses (Valdunciel, 2011). En origen, este tipo de urbanizaciones surgieron a partir de la creación de una junta de propietarios o bien a partir de un promotor que vendía las parcelas a propietarios que con el tiempo construían su vivienda en unas urbanizaciones que no disponían -y algunas no disponen todavía- de los equipamientos ni los servicios públicos necesarios. Actualmente, estas urbanizaciones han dejado de ser casi exclusivamente de segunda residencia para dar paso al establecimiento de población permanente siguiendo las dinámicas propias de la dispersión de la población urbana sobre el territorio. Según datos del INE del año 2000, el 14,8\% de la población de los municipios del área de estudio (unos 12.068 habitantes) estaban empadronados en urbanizaciones de estos municipios, pasando a representar el $17,3 \%$ en 2005 , y el $19,7 \%$ en 2010.

\section{METODOLOGÍA}

\subsection{Muestreo e instrumentación}

La información utilizada en este estudio fue obtenida a través de encuestas presenciales realizadas a los residentes de una muestra de viviendas unifamiliares del área de estudio. Todas las encuestas fueron realizadas en el verano de 2010, lo que hizo posible que, al ser período vacacional, se incluyera en la muestra a residentes temporales. Se calcula que aproximadamente existen 18.600 viviendas unifamiliares en el área de estudio, las cuales se distribuyen en 1577 secciones catastrales diferentes. Del total, una muestra de 172 secciones censales catastrales fue seleccionada al azar a fin de obtener una muestra total de 1650 hogares a encuestar. Debido al elevado número de segundas residencias, muchas de las cuales no estaban ocupadas en el momento de la recogida de datos, se seleccionó una muestra de gran tamaño para compensar la probable baja tasa de respuesta. El número final de viviendas encuestadas fue de 240, aunque sólo la información de 234 encuestas fue utilizada en los análisis posteriores debido a que 6 de las viviendas encuestadas no poseían ningún tipo de jardín.

La encuesta incluye una batería de preguntas relativas a tres bloques de información: a) el perfil sociodemográfico del encuestado y su familia, b) las características físicas de la casa y su exterior, incluyendo el jardín, y c) las actitudes y conductas hacia la conservación del agua y las preferencias en jardinería. Éste último bloque de información contiene un conjunto de preguntas que constan de 8 ítems tipo escalas de Likert, a las que el encuestado debe otorgar una puntuación que va de 1 (completamente en desacuerdo) a 5 (completamente de acuerdo). La mayoría de los ítems sobre las actitudes y comportamientos que se incluyeron en el cuestionario fueron extraídos, previa adaptación a la realidad del contexto objeto de estudio, de Syme et al. (2004). 


\subsection{Análisis de las encuestas}

\subsubsection{Análisis clúster}

A partir de un análisis clúster se obtuvo una clasificación empírica de las diferentes tipologías de jardín que se dan en las urbanizaciones de esta área de estudio. Para ello se combinaron los dos métodos tradicionales de aglomeración (jerárquicos y no jerárquicos) para aprovechar las ventajas metodologicas de cada uno de ellos. En primer lugar se utilizó el método de obtención de conglomerados jerárquicos de Ward para establecer el número de conglomerados (4 como solución óptima) y los perfiles de los centros de los conglomerados. Estos fueron utilizados como puntos de semilla iniciales de los conglomerados para obtener resultados menos sesgados utilizando el método no jerárquico k-means.

Las variables que se introdujeron para cumplir con los objetivos exploratorios de este análisis responden a la lógica de la configuración física del jardín en función de su composición vegetal, características morfológicas y otros elementos existentes. En concreto las variables introducidas fueron:

- \%_afrutales: porcentaje de la zona plantada de la parcela ocupada por árboles frutales,

- \%_otrosarb: porcentaje de la zona plantada de la parcela ocupada por otros árboles,

- \%_pcrasas: porcentaje de la zona plantada de la parcela ocupada por plantas crasas,

- \%_arbustos: porcentaje de la zona plantada de la parcela ocupada por arbustos,

- \%_flores: porcentaje de la zona plantada de la parcela ocupada por bancales de flores,

- \%_cesped: porcentaje de la zona plantada de la parcela ocupada por césped,

- \%_huerto: porcentaje de la zona plantada de la parcela ocupada por huerto,

- Sup_patio: superficie total de la parte exterior de la vivienda $\left(\mathrm{m}^{2}\right)$,

- Piscina: presencia de piscina de obra o prefabricada ( 0 ó 1).

Como tarea preparatoria del análisis cluster, se aplicó previamente un análisis factorial con el objetivo de identificar variables subyacentes o factores que pudiesen explicar la configuración de las correlaciones dentro de este conjunto de variables observadas, (Hair et al., 1999). Al final del proceso, del conjunto de 9 variables originales se extrajeron 5 factores que explicaban aproximadamente un $73,2 \%$ de la varianza. Sólo se tomaron aquellos factores con un autovalor superior a 1. Estos mismos factores fueron utilizados posteriormente para obtener los 4 conglomerados resultantes de aplicar la metodología de aglomeración (tabla 1).

Tabla 1. Resultado de los grupos obtenidos del análisis clúster

\begin{tabular}{|c|c|c|}
\hline Grupos & Frecuencia & Porcentaje (\%) \\
\hline 1 & 126 & 52,5 \\
\hline 2 & 47 & 19,6 \\
\hline 3 & 51 & 21,3 \\
\hline 4 & 10 & 4,2 \\
\hline $5^{*}$ & 6 & 2,5 \\
\hline Total & 240 & 100,0 \\
\hline \multicolumn{3}{|c|}{ *Exterior sin jardín. } \\
Fuente: elaboración propia
\end{tabular}

\subsubsection{Perfiles sociodemográficos según el tipo de jardín}

Con el objetivo de determinar cuáles de las variables sociodemográficas exploradas estaban significativamente relacionadas con la elección de un tipo u otro de jardín, en este estudio se llevó a cabo un análisis de regresión logística multinomial.

Los modelos de regresión logística son modelos estadísticos a través de los cuales se quiere conocer la relación entre una variable dependiente cualitativa (que puede ser dicotómica [regresión logística 
binaria o binomial] o poseer más de dos valores [regresión logística multinomial]) y una o más variables explicativas independientes, o covariables, ya sean cualitativas o cuantitativas. En esta clase de modelos, la ecuación inicial es de tipo exponencial, si bien su transformación logarítmica (logit) permite su uso como una función lineal. Los dos principales objetivos de este tipo de modelo son: 1) Poder cuantificar la importancia de la relación existente entre cada una de las covariables y la variable dependiente, lo cual lleva implícito también aclarar la existencia de interacción y confusión entre covariables respecto a la variable dependiente; y 2) Poder clasificar individuos dentro de las categorías (presente/ausente o 0 y 1 ) de la variable dependiente, según la probabilidad de que tenga que pertenecer a una de estas categorías dada la presencia de determinadas covariables.

La estimación de los coeficientes de regresión logística se realiza de forma totalmente diferente a la regresión lineal múltiple. La naturaleza no lineal de la transformación logística requiere un procedimiento de estimación de máxima verosimilitud (y no mediante el método de los mínimos cuadrados como en el caso de la regresión lineal), que se utiliza de forma iterativa para encontrar la estimación "más probable" de los coeficientes. Para estimar el modelo de regresión logística se ajustan los datos reales a la curva logística. Cuanto mejor se ajuste la nube de puntos a esta curva más definida será la correlación y, por tanto, mayor magnitud tendrá el valor de verosimilitud. El procedimiento de cálculo del coeficiente logístico compara la probabilidad de ocurrencia de un suceso con la probabilidad de que no suceda. Esta probabilidad recibe el nombre de la razón de disparidad (u odds ratio).

En este estudio el análisis estadístico sólo se aplicó a aquellas tipologías de jardín con un número suficiente de casos, como sucede en los grupos $1(n=126), 2(n=47)$ y $3(n=51)$. La tabla 2 muestra las variables sociodemográficas que se utilizaron en este análisis. Se calcularon los odds ratio para 3 contrastes separados: (a) grupo 2 frente al grupo 1, (b) grupo 3 frente a grupo 1 y (c) grupo 3 frente a grupo 2. El odds ratio de valor 1,0 indica que cuando las otras variables del modelo se mantienen constantes, la variable explicativa no produce ningún efecto (probabilidades iguales). Los coeficientes superiores a 1,0 indican un efecto positivo en (o un aumento de las probabilidades de) la elección de un tipo de jardín frente a otro; coeficientes inferiores a 1,0 indican un efecto negativo en (o la disminución de las probabilidades de) la elección de un tipo de jardín frente a otro.

Tabla 2. Variables sociodemográficas utilizadas para predecir la elección de la tipología de jardín.

\begin{tabular}{|c|c|c|}
\hline Nombre de la variable & Descripción & Unidad \\
\hline Edad & Edad del encuestado & Años \\
\hline Tamaño de la familia & Número de miembros de la familia & $\mathrm{N}^{\mathrm{o}}$ \\
\hline Niños entre 0-9 años & La presencia de niños entre 0-9 años de edad en el hogar & $\begin{array}{l}\text { Variable ficticia } \\
\left(\text { no = } 0, s_{1}^{\prime}=1\right)\end{array}$ \\
\hline Piscina & Presencia de piscina de obra & $\begin{array}{l}\text { Variable ficticia } \\
\left(\text { no }=0, s_{1}=1\right)\end{array}$ \\
\hline Tipo de ocupación residencial & $\begin{array}{l}\text { SBI: residencia secundaria con bajo índice de ocupación (menos de } 127 \text { días al } \\
\text { año) } \\
\text { SAI: residencia secundaria con alto índice de ocupación ( } 127 \text { días al año o más) } \\
\text { RP: residencia principal }\end{array}$ & Variable categórica \\
\hline Nivel educativo & $\begin{array}{l}\text { Sin estudios } \\
\text { Primer grado: Mínimo EGB } \\
\text { Segundo grado: Mínimo ESO } \\
\text { Tercer grado: Mínimo diplomatura }\end{array}$ & $\%$ \\
\hline Valor catastral & $\begin{array}{l}\text { Valor que un tasador oficial asigna a una propiedad y sirve de base o como } \\
\text { referencia en relación con determinadas actuaciones de las administraciones } \\
\text { públicas. Proxy de ingresos del hogar }\end{array}$ & Miles de Euros \\
\hline $\begin{array}{l}\text { Interés en la jardinería ( } \alpha \text { de } \\
\text { Cronbach }=0,81)\end{array}$ & $\begin{array}{l}\text { Tres elementos de tipo Likert calificados en una escala de cinco puntos que van } \\
\text { de } 1 \text { (totalmente en desacuerdo) al } 5 \text { (completamente de acuerdo): } \\
\text { IJ1: Me proporciona gran satisfacción dedicarme al jardín. } \\
\text { IJ2: La jardinería es una pérdida de tiempo. } \\
\text { IJ3: Cuidar mi jardín es una manera agradable de romper con mi rutina. } \\
\text { IJ4: No me gusta la jardinería. }\end{array}$ & $\begin{array}{l}\text { de } 1 \text { (totalmente } \\
\text { en desacuerdo) al } \\
5 \text { (completamente } \\
\text { de acuerdo) }\end{array}$ \\
\hline
\end{tabular}




\begin{tabular}{|c|c|c|}
\hline $\begin{array}{l}\text { Recreación en el jardín ( } \alpha \text { de } \\
\text { Cronbach }=0,65 \text { ) }\end{array}$ & $\begin{array}{l}\text { RJ1: Me gusta enseñar mi jardín a mis amigos y familiares. } \\
\text { RJ2: Paso buenos ratos en mi jardín. } \\
\text { RJ3: Los que viven en esta casa nunca hace uso del jardín. } \\
\text { RJ4: La parte exterior de la vivienda es un lugar ideal para celebrar cenas o } \\
\text { comidas. }\end{array}$ & $\begin{array}{c}\text { de } 1 \text { (totalmente } \\
\text { en desacuerdo) al } \\
5 \text { (completamente } \\
\text { de acuerdo) }\end{array}$ \\
\hline $\begin{array}{l}\text { Actitudes hacia el ahorro del } \\
\text { agua }(\alpha \text { de Cronbach }=0,63)\end{array}$ & $\begin{array}{l}\text { AAAl: Ahorrar agua requiere un esfuerzo que no merece la pena (puntuación } \\
\text { inversa). } \\
\text { AAA2: El agua del grifo es un recurso demasiado valioso para ser desperdiciado. } \\
\text { AAA3: Ahorrar agua es un deber que se tiene que cumplir incluso cuando no se } \\
\text { está en situación de sequía. }\end{array}$ & $\begin{array}{c}\text { de } 1 \text { (totalmente } \\
\text { en desacuerdo) al } \\
5 \text { (completamente } \\
\text { de acuerdo) }\end{array}$ \\
\hline
\end{tabular}

\section{RESULTADOS}

\subsection{Tipologías de jardín}

La tabla 3 reúne las características más remarcables de cada uno de los 4 tipos de jardín resultantes de la aplicación de la metodología descrita en el apartado anterior. La figura 2 muestra una recopilación con imágenes reales de jardines que coinciden con los resultados obtenidos tomadas en las diferentes urbanizaciones del área de estudio.

Clúster 1. Jardín ornamental. Es el más numeroso $(n=126)$. Se caracteriza por tener la mayor proporción media de plantas crasas (20\%), arbustos (35\%) y bancales de flores (26\%). Los otros tipos de vegetación tienen poco protagonismo. Representa el grupo con la menor superficie exterior $\left(699,04 \mathrm{~m}^{2}\right)$ y la menor presencia de piscinas (el 47,62 \% de las viviendas encuestadas no la tienen).

Clúster 2. Jardín con huerto $(n=47)$. La parte vegetada del exterior de la vivienda suele estar ocupada en más de la mitad de su superficie (59\%) por un huerto.

Clúster 3. Jardín con césped $(n=51)$. El césped es el elemento más destacado en este tipo de jardín llegando a ocupar una proporción media del 63\% del total de la superficie exterior de la vivienda. También cuenta con una elevada tasa de presencia de piscina $(72,6 \%)$.

Clúster 4. Jardín arbolado $(n=10)$. Los árboles, tanto frutales como ornamentales, ocupan el $51 \%$ y el 35\%, respectivamente, de la superficie del jardín. Es el tipo de jardín que domina en los exteriores de mayor superficie $\left(914,6 \mathrm{~m}^{2}\right)$.

Tabla 3. Características particulares de cada uno de los grupos resultantes del análisis clúster.

\begin{tabular}{|c|c|c|c|c|}
\hline Variable & Clúster 1 & Clúster 2 & Clúster 3 & Clúster 4 \\
\hline$\%$ afrutales & 0 & 0 & 0 & $\odot \bigcirc$ \\
\hline \%_otrosarb & 0 & 0 & 0 & $\odot \odot$ \\
\hline \%_pcrasas & ○ & 0 & 0 & 0 \\
\hline$\%$ \%arbustos & $\bigcirc \bigcirc$ & 0 & 0 & 0 \\
\hline \%_flores & $\odot \odot$ & 0 & 0 & 0 \\
\hline$\%$ \%cesped & 0 & 0 & $\bigcirc \bigcirc$ & 0 \\
\hline \%_huerto & 0 & $\bigcirc \bigcirc \bigcirc$ & 0 & 0 \\
\hline Piscina (\% si) & $\bigcirc \bigcirc$ & $\bigcirc \bigcirc$ & 0000 & $\bigcirc \bigcirc$ \\
\hline Sup_patio & 699,04 & 761,40 & 791,35 & 914,60 \\
\hline
\end{tabular}

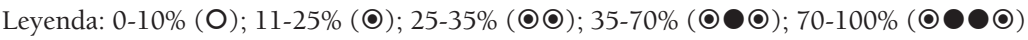

Fuente: elaboración propia. 
Figura 2. Ejemplos de jardín césped (parte superior izquierda), huerto (parte superior derecha), ornamental (parte inferior izquierda) y arbolado (parte inferior derecha).
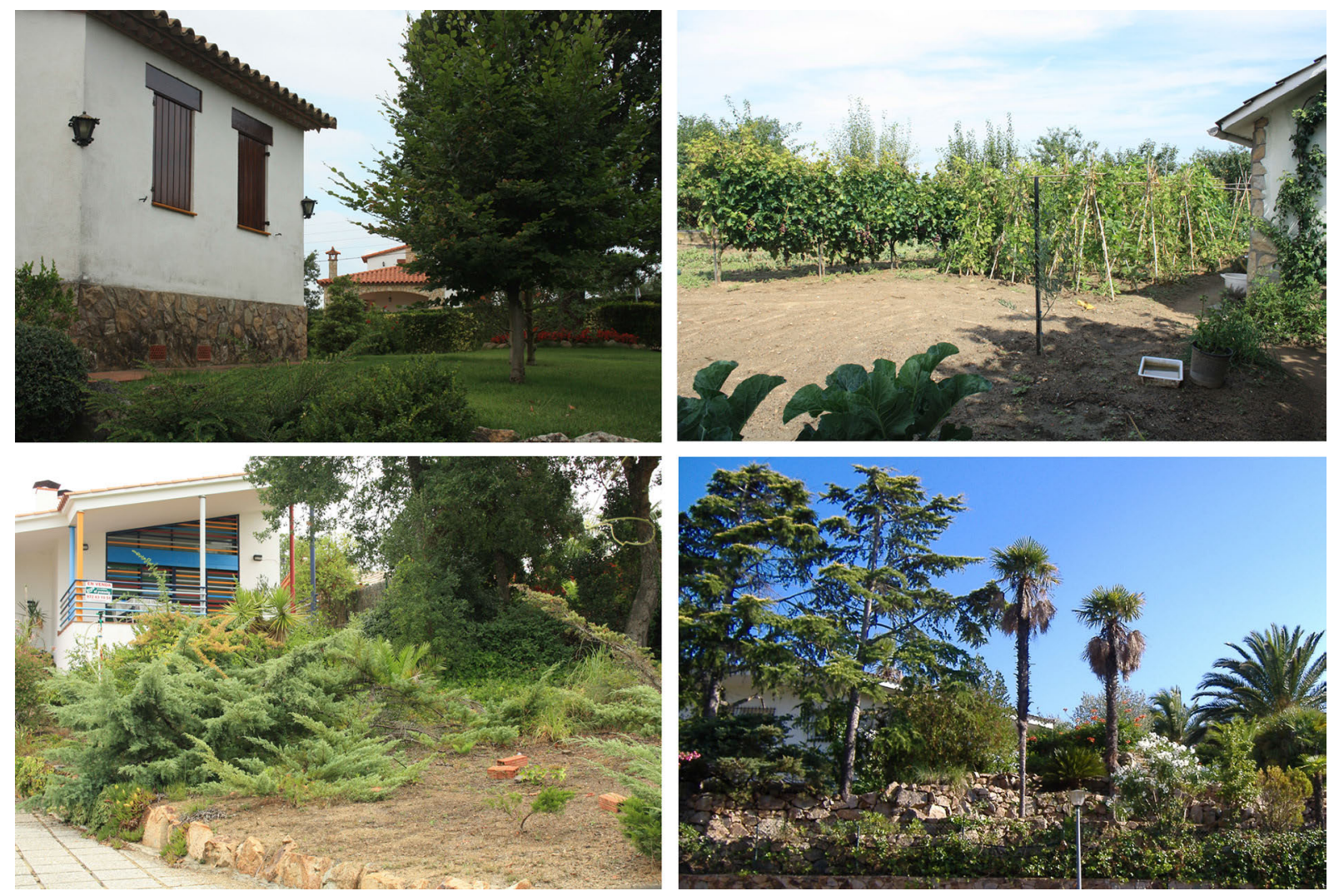

Fuente: elaboración propia.

\subsection{Perfiles sociodemográficos de los residentes}

El modelo de regresión logística multinomial explicó alrededor del 26\% de la varianza presente en la muestra (Tabla 4). Según los resultados, un tamaño del hogar superior demuestra contribuir a una mayor preferencia hacia un jardín con huerto en contraposición con un jardín ornamental. La adición de un miembro al hogar aumenta en un $45 \%$ las probabilidades de tener un jardín con huerto en comparación con un jardín ornamental, siempre que se mantengan constantes las otras variables. La presencia de niños en el hogar con edades comprendidas entre los 0 y los 9 años es, en cambio, una variable que no afecta significativamente la elección del tipo de jardín, al igual que la variable del valor catastral. La edad de la persona encuestada, contrariamente, parece afectar significativamente la elección de un jardín con césped por encima de un jardín con huerto de manera positiva (la variable tiene un odds ratio con valor 1,05). En cuanto a la variable "presencia de piscina", influye significativamente en la elección de un jardín con césped, en comparación tanto con el jardín ornamental como con el jardín con huerto. La presencia de piscina hace aumentar en un $62 \%$ la posibilidad de escoger un jardín con césped vs. un jardín ornamental, y un $67 \%$ la probabilidad de escoger un jardín con césped vs. un jardín con huerto. En cuanto al tipo de ocupación de la vivienda, los resultados sólo han mostrado una interacción significativa cuando se han comparado las categorías residencia principal (RP) y la residencia secundaria con tasa alta de ocupación (SAI). Poseer una residencia principal (en comparación a una residencia secundaria con alto índice de ocupación), según los resultados, hace aumentar un $80 \%$ la probabilidad de elección de un jardín con césped en lugar de un jardín ornamental, y un 81 \% la elección de un jardín con césped en lugar de un jardín con huerto. No tener estudios, en cambio, muestra favorecer en un $95 \%$ adicional de posibilidades la elección del jardín con huerto y la elección del jardín ornamental en lugar de un jardín con césped, en comparación con las personas encuestadas que tienen un nivel educativo de tercer grado. El resto de comparaciones de las categorías respectivas de la variable "nivel educativo" no parecen ser significativas. 
Las variables "interés en la jardinería" y "recreación en el jardín" no parecen repercutir en ninguna de las comparaciones de tipo de jardín, excepto en el caso de la comparación jardín con césped vs. jardín con huerto. El incremento de un punto de la variable "interés en la jardinería" hace un 52\% más plausible la elección de un jardín con huerto. En cambio, el incremento de un punto de la variable "recreación en la jardinería" hace aumentar unas 2,19 veces la posibilidad de elección de un jardín con césped. La variable "actitudes hacia el ahorro del agua", según los resultados, no influye de manera significativa en ninguna de las tres comparaciones.

Tabla 4. Razón de disparidad de los coeficientes de regresión logística multinomial.

\begin{tabular}{|l|c|c|c|}
\hline \multirow{2}{*}{ Variables explicativas } & Huerto / Ornamental & Césped / Ornamental & Césped / Huerto \\
\cline { 2 - 4 } & Exp. (b) & Exp. (b) & Exp. (b) \\
\hline Edad & 0,99 & 1,04 & $0,05^{*}$ \\
\hline Tamaño de la familia & $1,45^{*}$ & 1,24 & 0,58 \\
\hline $\begin{array}{l}\text { Niños entre 0-9 años ("sí" es la categoría de } \\
\text { referencia) }\end{array}$ & 1,16 & 0,67 & $0,33^{*}$ \\
\hline Piscina ("sí" es la categoría de referencia) & 1,14 & $0,38^{*}$ & 2,16 \\
\hline Ocupación residencial (PR es la categoría de & & & $0,19^{*}$ \\
referencia) & 0,61 & 1,31 & $0,05^{*}$ \\
SBI & 1,05 & $0,20^{*}$ & 0,69 \\
SAI & & & 1,50 \\
\hline Nivel educativo (Tercer grado es la categoría de & 1,14 & $0,05^{*}$ & 1,09 \\
referencia) & 0,65 & 0,45 & $0,48^{*}$ \\
Sin educación & 0,37 & 0,56 & $2,19^{*}$ \\
Primer grado & 1,09 & 1,19 & 1,78 \\
Segundo grado & 1,39 & 0,68 & 1,78 \\
\hline Valor catastral & 0,81 & 1,55 & \\
\hline Interés en la jardinería & 0,87 & & \\
\hline Recreación en el jardín & & & \\
\hline Actitudes de conservación del agua & & & \\
\hline
\end{tabular}

Nota: Chi-cuadrado $=60,79(\mathrm{df}=26, \mathrm{p}<0,000, \mathrm{n}=222)$. La $R^{2}$ de Cox y Snell es 0,240 $(\mathrm{p}<0,000)$

$\mathrm{p}<0,05^{*}$. Fuente: elaboración propia.

\section{DISCUSIÓN}

Este estudio ha permitido comprobar que no existe un patrón homogéneo de jardín en las urbanizaciones del sur de la comarca de La Selva sino todo lo contrario. El microcosmos social que representan estas urbanizaciones muestra una heterogeneidad interna que genera un mosaico de estilos de vida, y en consecuencia, de preferencias de jardín muy diversas. Con el objetivo de predecir el comportamiento en la elección del tipo de jardín basado en diversas variables sociodemográficas, en el presente estudio se llevó a cabo un modelo de regresión logística multinomial. Los resultados ofrecen una mejor comprensión de las relaciones que existen entre tales variables y el tipo de jardinería que practican los residentes en urbanizaciones.

Sorprendentemente, la presencia de niños en el hogar no condicionó significativamente la selección de ningún tipo de jardín en particular. Investigaciones anteriores habían demostrado la tendencia de las familias a preferir césped cuando se tienen niños pequeños (Yabiku et al., 2008). Sin embargo, en nuestro caso de estudio no siempre esta circunstancia es lo bastante condicionante cómo para ser asociada a la elección de este tipo de jardín (Hurd, 2006).

Según los resultados, un mayor tamaño del hogar favorece claramente la creación de un jardín con huerto cuando éste es comparado con el tipo de jardín ornamental. Un mayor número de personas en el hogar puede significar la necesidad de más alimentos o, simplemente, que en estos hogares los miembros 
de edad más avanzada, con una concepción del jardín como espacio para la recreación y/o producción de alimentos, optan por tener un huerto. En relación con la gestión del agua en los espacios residenciales, este hallazgo resulta interesante, dado que la tendencia general, según datos del Institut d'Estadística de Catalunya (IDESCAT), es que el tamaño de los hogares vaya disminuyendo en los próximos años (de 2,59 a 2,52 personas por hogar en Cataluña entre el año 2011 y 2021).

Con respecto a la variable edad, el estudio concluye que cuanto mayor es la edad del residente más aumenta su preferencia por el jardín con césped respecto a la opción del huerto. Van den Berg y Van Winsum-Westra (2010) también comprobaron que los propietarios de mayor edad optaban con más probabilidad por jardines con césped que no por aquellos más asilvestrados. Se debe tener en cuenta, en relación con esta variable, que se esperaba una mayor preferencia por el huerto en el caso de los residentes de edad avanzada puesto que las personas mayores, con un antecedente más rural, es más probable que destinen el jardín a la producción de alimentos (Head et al., 2004). Que se hayan incorporado al modelo variables como el nivel educativo, o el tipo de ocupación de la vivienda, puede haber provocado el cambio de signo de los coeficientes de esta variable. Se podría deducir que los residentes principales de mayor edad, o los residentes con un nivel educativo de tercer grado de mayor edad (categorías de referencia de estas dos variables), optan a menudo por un jardín con césped, en lugar de un huerto. En cambio, residentes en viviendas secundarias con una tasa alta de ocupación, y personas sin estudios (las dos categorías que agrupan los residentes de mayor edad) mostraron una tendencia positiva significativa a la implantación de un jardín con huerto.

Tal y cómo se había hipotizado, la presencia de una piscina está fuertemente vinculada con la tendencia a tener un jardín con césped, en comparación a las dos tipologías de jardín restantes. Este resultado demuestra que la idealización social de la casa con "piscina-césped", creada desde los medios de comunicación y las empresas inmobiliarias para mercantilizar el stock de vivienda, se plasma en la realidad del área de estudio

Con respecto a la tasa de ocupación de la casa, se quiso contrastar si los residentes en viviendas principales y secundarias se comportaban de manera diferente cuando se trataba de ajardinar sus hogares. Según se observa de los resultados obtenidos, no se ha detectado ninguna interacción entre estas variables y el comportamiento en relación al tipo de ajardinamiento. Sin embargo, los residentes en viviendas secundarias con un alto índice de ocupación mostraron un rechazo significativo por los jardines con césped (en beneficio del jardín ornamental y el jardín con huerto) en comparación con los residentes principales. A pesar de que se supone que los residentes principales presentan un mayor arraigo al lugar, Misetic (2006) y Stedman (2006) confirmaron que los individuos pueden desarrollar un fuerte vínculo e identificación con la ubicación de su segunda residencia. El fuerte arraigo al lugar de los residentes secundarios con alta tasa de ocupación puede contribuir a la práctica de jardines ornamentales y/o huertos. Este tipo de residentes suelen ser pre-jubilados o jubilados, con un nivel de ingresos medianobajo, que residen principalmente en el Área Metropolitana de Barcelona, pero que disfrutan gran parte del año (o casi todo el año) de su segunda residencia. Según se puede deducir de estos resultados, la tendencia a convertir residencias secundarias en principales en las urbanizaciones del área de estudio sólo tendría una elevada repercusión en las necesidades de riego de los jardines si se parte de una residencia secundaria con alto índice de ocupación.

El nivel educativo también mostró influir en la manera de ajardinar los hogares. Los residentes de mayor nivel educativo se mostraron significativamente más propensos a tener un jardín con césped en comparación con las personas encuestadas sin estudios. Sin embargo, las investigaciones realizadas por Hurd (2006) o Mustafa et al. (2010) para otros ámbitos de estudio demostraron que un mayor nivel educativo moderaba el deseo de poner césped y contribuía decisivamente a la elección de jardines con pocos requerimientos hídricos. No es nuevo que la población de menor nivel educativo se suela comportar de manera más pro-ambiental por lo que se refiere al ahorro del agua (De Oliver, 1999; Gilg y Barr, 2006).

El nivel de ingresos del hogar, indicado en este caso por el valor catastral, no mostró un favoritismo por jardines más mésicos como los de césped (Domene y Saurí, 2003) u ornamentales (Larsen y Harlan, 2006), teniendo en cuenta que este último tipo es lo más similar a lo que sería un jardín xérico. De hecho, este indicador no se relacionó significativamente con ningún tipo de jardín. En general, los exteriores de 
las viviendas en estas urbanizaciones tienen muros que esconden el jardín a los vecinos y transeuntes, difuminando parcialmente el rol social que en otras partes acostumbran a tener los jardines (Askew y McGirk, 2004).

Uno de los resultados más interesantes es el relacionado con la interacción entre las variables "interés en la jardinería" y "recreación en el jardín", y su significativa mediación a la hora de elegir un jardín con huerto o un jardín con césped. Syme et al. (2004) demostraron que ambos tipos de variables influían positivamente en un mayor consumo de agua para usos exteriores. Un alto interés en la jardinería influye positivamente en la elección del jardín con huerto, en comparación con el jardín con césped. Una valoración positiva del jardín como un elemento para la recreación muestra el efecto contrario. Lo que se demuestra con estos resultados, desde la óptica del impacto sobre los consumos de agua en el hogar, es que ambas variables, interés en la jardinería y recreación en el jardín, no afectan en la elección de un jardín más xérico o más mésico, debido a que tanto el jardín con césped como el huerto, se consideran mésicos.

Finalmente, la variable "actitudes hacia el ahorro del agua" no se relacionó significativamente con ninguno de los tipos de jardín contrastados en este estudio. A pesar de la hipótesis que un fortalecimiento de las actitudes hacia el ahorro del agua se debería asociar a una práctica más habitual de la jardinería de tipo xérico (Hurd, 2006), este resultado no se plasmó en este caso. Una explicación podría ser la escasa relación que existe entre las actitudes hacia el ahorro del agua y el comportamiento que se acaba manifestado en el consumo real de agua (De Oliver, 1999). Además, la preferencia por un determinado tipo de jardín ya sea pensando en ahorrar agua o no, a menudo no coincide con el tipo de jardín por el que se acaba optando debido al elevado coste económico que requiere hacer cambios significativos en el tipo de jardín (Larsen y Harlan, 2006).

\section{CONCLUSIONES}

Son diversos los estudios que se han centrado en examinar qué factores intervienen en los consumos de agua para usos domésticos en general, pero son todavía pocos los que se han interesado por aunar en los factores más influyentes en la elección de un tipo u otro del jardín. Este elemento de la vivienda, en ambientes como el mediterráneo, puede llegar a representar más del 50\% del total de consumo de agua del hogar.

El estudio que aquí se ha presentado ha podido comprobar que factores como el nivel educativo, la edad o el tipo de ocupación de la vivienda pueden resultar relevantes a la hora de elaborar estrategias de gestión en este ámbito. Los constructores y los compradores deben ser conscientes de los costes de mantenimiento de jardines mésicos en un clima mediterráneo. Las empresas inmobiliarias y los medios de comunicación pueden promover el ahorro de agua en las zonas residenciales suburbanas mediante la práctica de una mayor responsabilidad ambiental.

A menudo, en las campañas de ahorro de agua, se señala acertadamente los jardines con césped como los que más agua consumen, y se recomienda que habría que substituir su superficie por otros tapices vegetales u otros materiales. Sin embargo, en ninguno de los casos conocidos se considera la horticultura en el hogar y su relevante papel como consumidora de agua. Parecería adecuado establecer campañas de sensibilización en los usos del agua en las cuales se tuviera en cuenta también el elemento horticultura y la persona o personas que la practican. Los movimientos sociales que abogan por el consumo de productos locales y la concepción moderna de la práctica de la jardinería y/o la horticultura para usos terapéuticos, por ejemplo, representan un cambio hacia estilos de vida más saludables que contribuyen a la proliferación de huertos en los hogares. La actual crisis económica, que constriñe los presupuestos de las familias y puede aumentar su tamaño debido al retorno de antiguos miembros ya emancipados, también podría apoyar esta tendencia hacia jardines más productivos, por lo que estas campañas de educación ambiental deberían contemplar la reutilización de agua (como la sobrante de la piscina, etc.), o la recolección de agua de lluvia como sistemas a aplicar en el riego de estos jardines y huertos.

Hay que transmitir, desde las autoridades gestoras del agua y hacia la ciudadanía, que la preferencia por jardines privados con un consumo elevado de agua deriva en un alto coste ambiental en términos de 
riego cada vez menos asumible en un contexto que se prevé que empeore debido a la creciente severidad climática en términos de reducción de las precipitaciones (Llebot, 2010). El césped artificial, u otros tipos de tapices, pueden muy bien sustituir las funciones estéticas o prácticas que se atribuyen al césped. El concepto de ordenación estética que transmite el césped tendría que vincularse a otras opciones más adecuadas en el ámbito mediterráneo, como la plantación de especies autóctonas. Este cambio también favorecería la presencia de especies animales autóctonas y ayudaría a reforzar las experiencias sensoriales de contacto con la naturaleza (Bhatti y Andrew, 2004; Fuller et al., 2007).

\section{BIBLIOGRAFÍA}

ASKEW, L. E. y MCGUIRK, P. M. (2004): "Watering the suburbs: distinction, conformity and the suburban garden", en Australian Geographer, no 35, pp. 17-37.

BHATTI, M. y CHURCH, A. (2000): "I never promised you a rose garden: gender, leisure and homemaking" en, Leisure Studies, nº 19, pp. 183-197.

CLAYTON, S. (2007): "Domesticated nature: Motivations for gardening and perceptions of environmental impact", en Journal of Environmental Psychology, n 27, pp. 215-224.

COLLINS, R., KRISTENSEN, P., y THYSSEN, N. (2009): Water resources across Europe - confronting water scarcity and drought. European Environmental Agency EEA Report series. N. 2/2009. ISSN 1725-9177. EEA. Copenhagen, $55 \mathrm{p}$.

DAVIDSON, D. J., y FREUDENBURG, W. R. (1996): "Gender and environmental risk concerns a review and analysis of available research", en Environment and behavior, $\mathrm{n}^{\circ}$ 28(3), pp. 302-339.

DE OLIVER, M. (1999): "Attitudes and inaction: A case study of the manifest demographics of urban water conservation", en Environment and Behavior, $\mathrm{n}^{\circ}$ 31, pp. 372-394.

DOMENE, E. y SAURÍ, D. (2003): "Modelos urbanos y consumo de agua: el riego de jardines privados en la región metropolitana de Barcelona", en Investigaciones Geográficas, nº 32, pp. 5-17.

DOMENE, E., y SAURÍ, D. (2006): "Urbanisation and water consumption: Influencing factors in the metropolitan region of Barcelona", en Urban Studies, no 43(9), pp. 1605-1623

DTS (2010) [Dir.]: Platerritorial parcial de les Comarques Gironines. Departament de Territori i Sostenibilitat.

EEA - EUROPEAN COMMISSION (2006) [Dir.]: Urban Sprawl in Europe: The ignored challenge. Report Number 10/2006, European Environmental Agency. <http://www.eea.europa.eu/publications/eea report 2006 10>, [consulta: 10/10/2009]

EEA - EUROPEAN COMMISSION (2009) [Dir.]: Water resources across Europe-confronting water scarcity and drought. Report Number 02/2009, European Environmental Agency. < http://www.eea.europa.eu/ publications/water-resources-across-europe>, [consulta: 15/02/2010]

EEA - EUROPEAN COMMISSION (2012): A Blueprint to Safeguard Europe's Water Resources. European Commission. Brussels <http://ec.europa.eu/environment/water/blueprint/>, [consulta: 15/11/2013]

FULLER, R. A., IRVINE, K. N., DEVINE-WRIGHT, P., WARREN, P. H., y GASTON, K. J. (2007): "Psychological benefits of greenspace increase with biodiversity" en, Biology letters, $\mathrm{n}^{\circ}$ 3(4), pp. 390-394.

GARCIA, X., RIBAS, A., LLAUSÀS, A., y SAURÍ, D. (2013a): "Socio-demographic profiles in suburban developments: Implications for water-related attitudes and behaviors along the Mediterranean coast", en, Applied Geography, no 41, pp. 46-54.

GARCIA, X., MURO, M., RIBAS, A., LLAUSÀS, A., JEFFREY, P. y SAURÍ, D. (2013b): "Attitudes and behaviours towards water conservation on the Mediterranean coast: the role of socio-demographic and place-attachment factors" en, Water International, $\mathrm{n}^{\circ} 38$, pp. 1-14.

GILG, A. y BARR, S. (2006): "Behavioural attitudes towards water saving? Evidence from a study of environmental actions" en, Ecological Economics, n 57 , pp. 400-414. 
GREGORY, G.D., DI y LEO M.(2003): "Repeated behaviour and environmental psychology: the role of personal involvement and habit formation in explaining water consumption" en, Journal of Applied Social Psychology, no 33, pp. 1261-1296.

HAIR, J.F., ANDERSON,R.E., TATHAM, R.L. y BLACK W.C. (1999): Análisis multivariante. Prentice. Madrid.

HEAD, L., MUIR, P. y HAMPEL, E. (2004): Australian backyard gardens and the journey of migration", en, Geographical Review, no 94(3), pp. 326-347.

HESS, T. M. (2001): "Ageing-related influences on personal need for structure", en International Journal of Behavioral Development, $\mathrm{n}^{\circ}$ 25(6), pp. 482-490.

HOPE, D., GRIES, C., ZHU, W., FAGAN, W., REDMAN, C.L., GRIMM, N.B., et al. (2003): "Socioeconomics drive urban plant diversity", en Proceedings of the National Academy of Science, n 100, pp. 8788-8792.

HURD, B.H. (2006): "Water conservation and residential landscapes: household preferences, household choices", en Journal of Agricultural and Resource Economics, n 31, pp. 173-192.

KAPLAN, R. (1973): "Some psycholojgical benefits of gardening", en Environment and Behavior, n 5, pp. 145-162.

KIESLING, F. M., y MANNING, C. M. (2010): "How green is your thumb? Environmental gardening identity and ecological gardening practices", en Journal of Environmental Psychology, n 30(3), pp. 315-327.

LARSEN, L. y HARLAN S.L. (2006): "Desert dreamscapes. Residential landscape preference and behaviour", en Landscape and Urban Planning, n' 78, pp. 85-100.

LARSON, K.L., CASAGRANDE, D., HARLAN, S.L., y YABIKU, S.T. (2009): Residents' yard choices and rationales in a desert city: Social priorities, ecological impacts, and decision tradeoffs", en Environmental Management, $\mathrm{n}^{\circ} 44$, pp. 921-937.

LARSON, K. L., COOK, E., STRAWHACKER, C., y HALL, S. J. (2010): "The influence of diverse values, ecological structure, and geographic context on residents' multifaceted landscaping decisions2, en Human Ecology, n 38(6), pp. 747-761.

LLEBOT, J.E. (ed.) (2010): Segon informe sobre el canvi climatic a Catalunya. Consell Assessor per al Desenvolupament Sostenible. Barcelona.

MISETIC, A. (2006): "Place attachment and second home", en Drustvena Istrazivanja, no 15, pp. $27-42$.

MUSTAFA, D., SMUCKER, T. A., GINN, F., JOHNS, R., y CONNELY, S. (2010): "Xeriscape people and the cultural politics of turfgrass transformation", en Environment and Planning D: Society and Space, $\mathrm{n}^{\mathrm{o}}$ 28(4), pp. 600-617.

NEL·LO, O. (2001): Ciutat de ciutats: reflexions sobre el procés d’urbanització a Catalunya, Barcelona, Empúries, 123 p.

SIME, J. (1993): What makes a house a home: the garden, En BULOS, M. y TEYMUR, N. Housing: Design, Research, Education, Aldershot, Avebury, pp. 239-254.

SAURÍ, D. (2003): "Lights and shadows of urban water demand management: The case of the Metropolitan Region of Barcelona", en European Planning Studies, n 11, pp. 229-243.

STEDMAN, R.C. (2006): "Understanding place attachment among second home owners", en American Behavioral Scientist, $\mathrm{n}^{\circ}$ 50, pp. 187-205.

ST. HILAIRE, R., SPINTI, J.E., VANLEEUWEN, D. y SMITH, C. (2003): Landscape Preferences and Attitudes Toward Water Conservation: A Public Opinion Survey of Homeowners in Las Cruces, New Mexico. Agricultural Experiment Station. Research Report 750.

SYME, G.J., KANTOLA S.J. y THOMAS J.F. (1980): Water resources and the quarter acre block. En THORNE R. y ARDEN, S., People and the Man Made Environment, Sydney, University of Sydney, pp. 192-201. 
SYME, G.J., SHAO, Q., PO, M. y CAMPBELL, E. (2004): "Predicting and understanding home garden water use", en Landscape and Urban Planning, $\mathrm{n}^{\circ}$ 68, pp. 121-128.

THOMAS, J.S. y DURHAM, B. (2003): "Integrated Water Resource Management: looking at the whole picture", en Desalination, $\mathrm{n}^{\circ} 1561$, pp. 21-28.

VALDUNCIEL, J. (2011): Paisatge i models urbans contemporanis. Les Comarques Gironines (1979-2006): del desarrollismo a la globalització [en línea Girona: Universitat de Girona. Institut de Medi Ambient. Recuperado 10 desembre 2011, des de http://www.tdx.cat/handle/10803/32044

VAN DEN BERG, A. E., y VAN WINSUM-WESTRA, M. (2010): "Manicured, romantic, or wild? The relation between need for structure and preferences for garden styles", en Urban forestry \& urban greening, $\mathrm{n}^{\circ}$ 9(3), pp. 179-186.

YABIKU, S.T., CASAGRANDE, D.G. y FARLEY-METZGER, E. (2008): "Preferences for landscape choice in a Southwestern desert city", en Environment And Behavior, n 40, pp. 382-400.

ZMYSLONY, J. y GAGNON, D. (2000): Path analysis of spatial predictors of front-yard landscape in an anthropogenic environment", en Landscape ecology, n 15(4), pp. 357-371. 